\title{
Analysis of Extraction Algorithm for Visual Navigation of Farm Robots Based on Dark Primary Colors
}

\author{
Jin Wang, College of Art, Hebei Agricultural University, Baoding, China \\ Yifei Cui, College of Art, Hebei Agricultural University, Baoding, China \\ Hao Wang, College of Art, Hebei Agricultural University, Baoding, China \\ Mohammad Ikbal, Lovely Professional University, Jalandhar, India \\ iD https://orcid.org/0000-0001-6148-1555
}

Mohammad Usama, Sunway University, Malaysia

\begin{abstract}
In order to quickly extract the visual navigation line of farmland robot, an extraction algorithm for dark primary agricultural machinery is proposed. The application of dark primary color principle in new farmland is made clearer by gray scale method, and the soil and crops are obviously separated, and the image processing technology of visual navigation line image of farmland is realized. In binary filtering of gray scale images, the maximum interclass variance method and morphological method are used respectively. The researchers use vertical projection method and least square method to the farmland interval extracted by navigation line. The farmland that needs the guide line image will be accurately located. It is found that the visual navigation extraction algorithm of farmland robot is widely used in the image extraction of navigation lines of various farmland roads and scenes compared with the traditional gray scale algorithm. Image processing has the advantages of clearer image processing.
\end{abstract}

\section{KEYWORDS}

Agricultural Machinery, Dark Primary Color, Farmland Environment, Machine Vision, Navigation Line

\section{INTRODUCTION}

With the stepping up of agricultural modernization, agricultural production is increasingly developing towards intelligence and precision, and the research and application of agricultural robots are becoming more and more popular (Wolfert et al., 2014). China's agricultural production activities are not sufficiently mechanized and intelligent and there is still a big gap with developed countries such as Europe and America (Welte, 2016). At present, research on automatic navigation of agricultural machinery mainly focuses on machine vision and Global Positioning System (GPS) navigation. Compared with GPS, machine vision has higher cost performance, richer detailed information, and more accurate local positioning. The illumination changes dramatically in the complex field 
environment, which has a great influence on the extraction of navigation lines. For natural light, researchers have done more research on color space, which can effectively reduce the impact of light on image processing (Sharma, A., et al., 2017). In the field of image processing, there are many shaping algorithms for image segmentation and separation of background and foreground. However, due to the limitation of speed and accuracy, a single image segmentation algorithm cannot fully meet the requirements of real-time and accuracy of farmland robot(Guo et al., 2018). At present, there are mainly Hough transform, LSD algorithm, least square method, and so on in the detection of crop line. The Hough transform method has a large amount of data and long processing time(Huang et al., 2018). LSD algorithm has the poor global performance. (Sharma, A., et al., 2019) The least-square solution of the matrix form will have no inverse matrix (De Wagter et al., 2018).

To sum up, the existing methods cannot be applied to crop navigation in a non-green environment, such as farmland in the farming period, newly sprouting seedling farmland, and crop farmland with low and mature straw, etc... (Dutta et al., 2014). Moreover, the linear scanning method is greatly affected by the image segmentation effect and other factors, which cannot meet the stability requirements(Zhao, 2019).

To quickly extract the agricultural machinery and visual navigation line, this paper proposes a navigation line extraction algorithm based on a dark color, the algorithm using the dark color to gray-scale transformation, then the image using the morphological filter and the between-cluster variance of gray image segmentation method (Otsu) filter, and then vertical projection method is used to extract the interesting region, the last use of interested area set point least-square fitting of navigation line. Otsu's method is an adaptive thresholding way for binarization in image processing. By going through all possible threshold values (from 0 to 255), it can find the optimal threshold value of the input image

To provide a navigation line extraction algorithm based on a dark color, the algorithm using the dark color to gray-scale transformation. The article is thus organized in the following order. Literature review of various techniques and algorithm are detailed in Section 2. Section 3 discusses the image preprocessing and segmentation with simulated and tested results. A comparison of different methods with the present structure is also conducted to shed light on the advantages of the proposed method. Finally, the manuscript is concluded in Section 4.

\section{LITERATURE REVIEW}

In 2000, Torii, et al., Briefly explains the numerous applications of image sensors, soft computing, fuzzy control, and cooperative performance in agricultural robots. Many observe by producers such as Kubota and Mitsubishi are concisely described. In 2006, Pedersen et al., studies various crop scouting, weeding, and grass cutting robots. Analyses the economic benefits of using robots over conventional grass cutting machines. In 2010, Cohen et al., studied navigation, agricultural procedures, navigation, and sensor systems along with their upcoming promises. In 2015, Tarannum et al. discuss various robot operations; land preparation, soil observation, seeding \& planting, plant observation, harvesting, and picking fruit. Garcia-Vico et al. proposed a new pan-sharpening algorithm that estimates the extracted details and injection gain utilizing significance analysis and the Gaussian mixture model. This method is applied to four reliable general sharpening methods based on CS and compared with other latest general sharpening methods.

For agricultural robot navigation control, Feng et al. used a proportional Integral derivative (PID) control method for accurate alignment of the wheat fertilizer applicator. Zhang et al. [14] designed course and lateral controllers based on PID for autonomous navigation of agricultural vehicles. After the system is stabilized, the tracking accuracy of the robot was in centimeters. However, the control parameters of the PID controller are difficult to be set in the complex environment of farmland. Zhang et al. proposed a linear tracking method based on fuzzy control. Simulation and experiment results showed that the paddy field weeding robot was able to track the road accurately. Meng et 
al.used a fuzzy logic controller to control a weeding implement robot in the agricultural field. The experimental results showed that the control system has good dynamic performance at three different speeds. Based on the visual navigation system, Tang et al.proposed a serial Back Propagation (BP) neural network control algorithm, this system can achieve high accuracy with the maximum position deviation of $0.069 \mathrm{~m}$ Through various algorithms for taking out navigation lines from orchards that have been reported in the above, technical problems still exist. Because of the tree shape, planting pattern. Experimental results show that the improved algorithm has better performance than the original one. Also, our method has achieved comparable or better performance than other advanced methods. Ye et al., proposed a human motion analysis algorithm based on bone extraction and RGBD camera dynamic time warping. Firstly, to solve the problem of limited two-dimensional spatial information, an RGBD camera is used to obtain three-dimensional spatial information of the human body. Then, in the feature extraction process, eleven skeleton points are selected from the depth image to calculate the relative space distance, which significantly reduces the computational complexity. Also, a dynamic time warping optimization algorithm is proposed to reduce the adverse effects of time difference. Finally, the motion analysis of the human body is studied. Experimental results show that this method can effectively identify single and double actions. Huang et al. proposed an algorithm simulation method based on the secondary development of Unity 3D software. In this method, a 3d robot model, virtual joint, and virtual sensors are imported to create a virtual robot prototype quickly, and an algorithm script is used for navigation simulation in a virtual environment. Firstly, the script of the virtual revolute joint, virtual laser radar sensor, and terrain environment was written. Secondly, the $\mathrm{A}^{*}$ algorithm is improved for the navigation of unknown 3D space. Thirdly, taking the Mecanum wheel mobile robot as an example, the 3D robot model was imported into Unity3D, and virtual joints, sensors, and navigation algorithm scripts were added to the model. Then, a virtual prototype is used to simulate navigation in static and dynamic environments. Finally, the navigation test of the physical robot is carried out in the physical environment, and the test trajectory is compared with the simulation trajectory. Simulation and test results verify the algorithm simulation method based on the secondary development of Unity3d software, which shows that the method is feasible, efficient, and flexible.

\section{IMAGE PREPROCESSING AND SEGMENTATION}

The application technology of my country's intelligent navigation technology in agricultural fields is becoming more and more mature. In various farmland scenes, image processing technology has been able to describe the landform features of the field scenes more accurately and quickly. Dong and Itoh, 2012). In the process of a large amount of data and information processing, it is found that due to the complex crops grown in farmland and the changeable terrain, the visual navigation line of gray-scale processing cannot adapt to image processing and segmentation(Cole et al., 2012). To perform image processing and effective segmentation for the key observation farmland, it is necessary to accelerate the extraction speed of the farmland navigation line information. Compared with the gray-scale processing technology, the image preprocessing technology of the farmland navigation line clearly distinguishes the soil and crops(Cooper et al., 2009). Open, not affected by day or night light, but when the crop is not green, this method loses its effect. Binary image refers to the image formed by the method of linear scanning after the soil is separated from farming. Based on the triangle of equal area, it is divided into two parts for scanning, and according to the same step length, the characteristics of the crop are passed. Perform image scanning work.

\subsection{Grayscale of Dark Primary Colors of Farmland Images}

When imaging farmland navigation lines, the flight path in the sky is generally not captured. in the image. Most of the crops in the farmland are bright colors, such as green, and only a few crops show dim colors. Therefore, in the farmland image based on the principle of dark primary color, if the color of the crop surface is brighter, or the color of the crop surface is dim, any channel value of the 
three channels of RGB. The channel value formed on the surface of the object is very small, and the dark primary color after farmland imaging the image is darker. Directly cause the dark primary color image of the soil and the dark primary color image of crops, one of them must be dimmed gray. This is the dark primary color principle of the grayscale of the farmland image. It is generally considered that the color is composed of three primary colors: blue, red, and green. In this experiment, the three primary colors are assumed to be RGB (r, g, b). These three primary colors are calculated using the rule of three primary colors. Therefore, when gray is used to represent three-point values, a new color is formed and the gray value of the point is obtained, that is, RGB (gray, gray, gray)(Ye et al., 2018). The visual navigation line of agricultural robots can be widely used in agricultural production(Dutta et al., 2014). Researchers have processed the image of the farmland navigation line on grayscale and dark primary color principle to make the scope of application more Broadly, this process is defined as "the image's dark primary color gradation processing" (El Bilali and Allahyari, 2018).

First: The researcher enters the navigation line image "I" in the selected experimental field, and digitally defines the principle of the dark channel, where the expression of each pixel in the dark channel is:

$$
\mathrm{I}^{\mathrm{dark}}(x, y)=\min _{\mathrm{c} \in[r, g, b]} I^{c}(x, y)
$$

In the above formula, (x, y) represents the pixel coordinates of the color channel in $I^{c}(x, y)$, and $(x, y)$ in $I^{d a r k}(x, y)$ represents the pixel coordinates of the person in the dark primary color. The three color channels in the above formula are compared in numerical value, and the three color channels are marked as $I_{r}(x, y), I_{g}(x, y), I_{b}(x, y)$. All pixels in I are compared according to the gray value of each pixel in the formula (1), and the final result is the dark primary color grayscale image value $I^{d a r k}$. With the help of Matlab (R2010b) software, to test the performance of the dark primary color grayscale method, and evaluate the image, compare the calculation time of the $2 \mathrm{G}-\mathrm{R}-\mathrm{B}$ super green model algorithm and the dark primary color grayscale image, and unified the performance of histogram characteristics. As shown in figure 1, (b) and (c) correspond to the 2G-R-B super-green crop field image, and (a) corresponds to the grayscale image. (a) It is observed from Figure 1(a) is converted to (b) and (c) is with conversion time values $0.532 \mathrm{~s}$ and $4.267 \mathrm{~s}$ respectively. In the histogram in Figure 1(b), we can see that the peaks and valleys of the farmland are more obvious. Compared with the histogram in Figure 1(c), the threshold value segmentation is easier to perform. It can be seen from the image of the grayscale conversion result in Figure 1 that the obvious distinction between the soil and green plants in the farmland can be completed by using the dark primary color grayscale image technology. At the same time, it can be seen that the 2G-R-B super green model algorithm is used.

The grayscale image of the farmland can also distinguish the farmland soil and green plants. But the outline of the green plant part is fuzzy and not clears enough, which greatly affects the feedback of the original image information of the farmland. Therefore, the $2 \mathrm{~g}-\mathrm{r}-\mathrm{b}$ ultra-green model algorithm

Figure 1. Grayscale conversion result (a) original image; (b) grayscale images of dark primary colors ;(c) 2 G - R - B grayscale

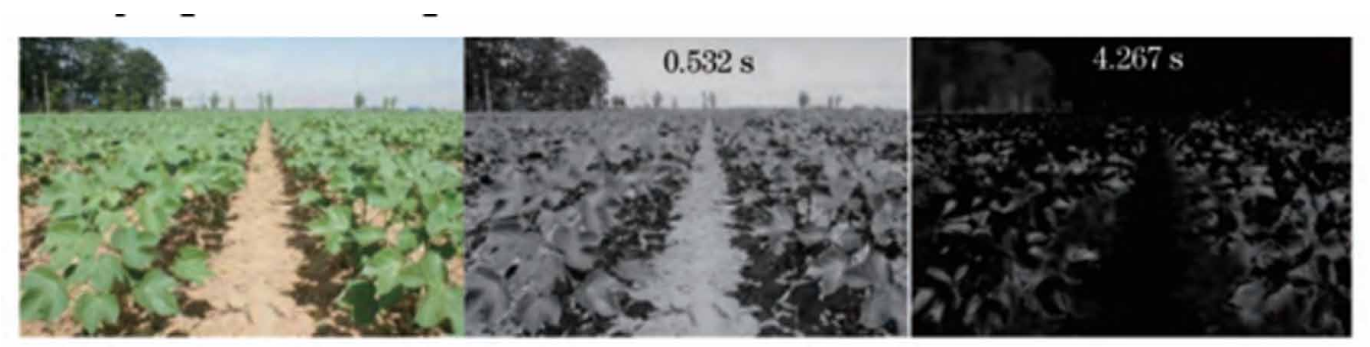


is slower than the dark primary grayscale image processing method, and it is not conducive to the distinction between soil and green plants. In daily life, there are not only green farmland environments but also farmland environments for many non-green crops, so that the application and promotion of dark primary color grayscale processing images in non-green farmland environments are also more important.

As shown in the following figure 2, it is a typical image feature of a non-green farmland environment. Figure 2(b) is the image of figure 2(a) based on the dark primary color grayscale processing. When the dark primary color grayscale processing transformation is performed on the images in Figure 2(a) and Figure 2(b), the 2g-RB super green model algorithm. In the green model conversion process, it loses its utility, as shown in Figure 2(c). It can be seen that when figure 2(a) is converted into a figure 2(b) and figure 2(c), the conversion time is $0.956 \mathrm{~s}$ and $2.827 \mathrm{~s}$ respectively. The wave crests and troughs in figure 2(b) are more obvious. Therefore, when using the gray conversion method of the dark primary color, it is necessary to use different environments according to local conditions and to meet the applicability and timeliness of the navigation line image in the farmland.

\subsection{Image Segmentation Based on Morphological Filtering and Otsu Method}

When the navigation line image is $I^{\text {dark }}$, the disc type uses a structure with a radius $(r)$ of 3 pixel to be corroded. Secondly, the navigation line image $I^{\text {dark }}$ is filtered at a window radius of 3 pixel*3pixel. Finally, the disk-shaped structure is expanded, and the $I_{f}$ image after the dark primary color grayscale is filtered. The dark primary color gradation processing in this process is processed in the order of "corrosion-filtering-expansion", which greatly reduces the potential interference factors in the shooting process.

After the above processing, the Otsu method is used to binarized the image. It can be seen from Figure 1 that after the obtained image is binarized, it is a segmentation method using threshold values, based on dark primary color grayscale image processing (Pavan W, Fraisse CW, Peres NA, 2011). Due to illumination, various environments, and different display brightness, farmland images will have large differences between the shooting target and background. Therefore, for the above situation, the global adaptive threshold value $\mathrm{K}$ is used to segment the dark primary color image. Its formula is expressed as(Zhao, 2019):

$$
\left\{\begin{array}{l}
\mu=\lambda_{0} \times \mu_{0}+\lambda_{1} \times \mu_{1} \\
\delta^{2}(\mathrm{k})=\lambda_{0} \times\left(\mu-\mu_{0}\right)^{2}+\lambda_{1} \times\left(\mu_{1}-\mu_{0}\right)^{2} \\
\delta^{2}\left(\mathrm{k}_{0}\right)=\max \left\{\left(\delta^{2(\mathrm{k})}\right)\right\}
\end{array}\right.
$$

Figure 2. Grayscale conversion result of dark primary color (a) original image;(b) grayscale images of dark primary colors;(c) $2 g-r-b$ grayscale;(d) gray histogram of dark primary colors;(e) $2 g-r-b$ gray histogram
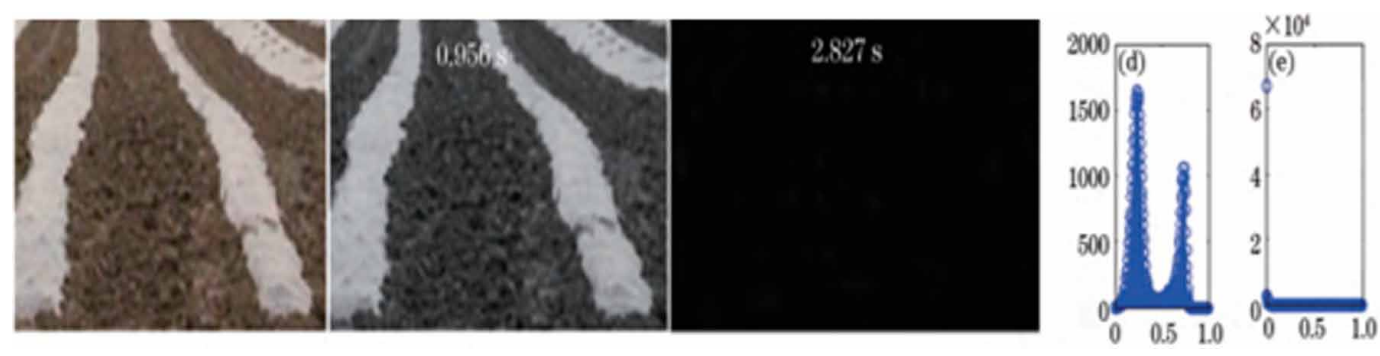
In the above formula, $\mu, \lambda_{0}$, and $\lambda_{1}$ is expressed as the total average grayscale of the image, the proportion of the foreground, and the proportion of the background, respectively. $\delta^{2}(k), k_{0}, \mu_{0,}$ and $\mu_{1}$ represent the variance of the foreground and the background, the optimal threshold, the average value of the foreground grayscale, and the average value of the background grayscale respectively (Vermesan and Friess, 2016). The binary image $I_{b c}$ can be obtained by judging the if grayscale image according to each pixel in the equation (3) through the following formula (Ntaliani and Costopoulou, 2013):

$\mathrm{I}_{\mathrm{bc}}(x, y)=\left\{\begin{array}{l}0, \mathrm{I}_{\mathrm{f}}(\mathrm{x}, \mathrm{y}) \prec \mathrm{k}_{0} \\ 1, \text { else }\end{array}\right.$

To remove the noise, lack of plants and seedlings, and granular noise in the binary image, the binary image is filtered according to the characteristics of the experimental field and the morphological method used, which can remove the binary image interference factors(Elsheakh et al., 2009). The disc-shaped structure with a radius of 5 pixels is selected for processing, and the final image after $I_{b}$ processing is obtained (Wolfert et al., 2014). It uses morphological closed operation to process the structure of 5 pixel $\times 1$ pixel (De Wagter et al., 2018;Yasuda, Y. D. et al.2020). This not only greatly improves the stability of the overall calculation method, but it is also easy to remove the fuzzy parts in the binary image, and fully express the farmland area that needs to be detected through the longitudinal structure, which greatly covers the holes in the field crop background (El Bilali and Allahyari, 2018).

\section{CENTER NAVIGATION LINE EXTRACTION BASED ON THE VERTICAL PROJECTION METHOD}

The following figure 3 is the binary image of the farm robot in the process of extracting the guidance line. Usually, the process of extracting images in the navigation line is mainly divided into three steps:

Step1: Use vertical projection technology (a1) to extract the image of the area to be extracted in the farmland (a2);

Step2: After obtaining the navigation positioning point, as shown in Figure (b), Perform point-toimage labeling;

Step3: Extract the area that needs to be extracted in the farmland based on the least square method, and synthesize the navigation line image, as shown in Figure (c) below.

\subsection{Extraction of Region of Interest}

Usually, when using agricultural machinery, the front direction is the shooting direction of the camera. The camera tool used is consistent with the field of view in the driving direction of the agricultural

Figure 3. Extraction process of binary image navigation line (a1\&a2) area of interest ;(b) registration point ;(c) navigation line

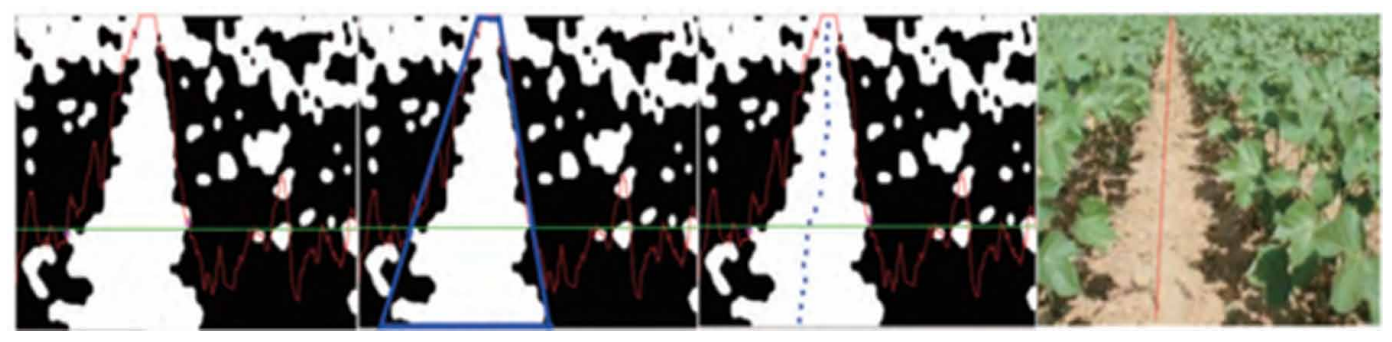


machinery. The area between the ridges is generally used as the navigation area of the camera during driving(Guo et al., 2018). In the binary image, the crops are marked as black areas, the soil between the ridges and the blank areas between the crop plants are marked as white areas, and the white marked areas are projected by the vertical image method. According to this feature, the agricultural machinery drives to get the navigation line of the area you want to extract(Zhu et al., 2019).

\subsection{Navigation Anchor Point Extraction}

To determine the middle positioning point of the navigation interval, the binary image needs to be divided into $n$ different horizontal images (Tilman et al., 2011). In this process, to reduce the number of calculations, the relative horizontal pixels should not be set too much. If the size of the image taken is $\mathrm{M}$ pixel $\times \mathrm{N}$ pixel(Tien, 2013), perform vertical projection on the obtained horizontal image. To find the highest area position in the vertical image, select an intermediate value from many horizontal images, and calculate the abscissa in these areas Take the average value so that you can prepare to determine the navigation anchor point. (Xindong Wu et al., 2014).

\section{EXPERIMENTAL RESULTS AND ANALYSIS}

Through Matlab2010 (b) software, a $3 \mathrm{GHz}$ PC is used for simulation experiments under the Windows 7 operating system. The pixels between 680 pixel $\times 450$ pixels and 500 pixel $\times 300$ pixels are used for comparison experiments. To prevent the difference of cameras from causing different specifications when shooting images on the navigation line (Vermesan and Friess, 2016). Use different types of images to compare farmland experiments, and use non-green crop images and green crop images in the experimental field as references.

The applicability and influencing elements of the green and non-green test fields are tested by image processing methods. Figure 4 shows the navigation line images in different green test field environments. Figure 5 is the result of extracting the navigation line of non-green farmland under different environments. It can be seen that the above-mentioned various algorithms can make accurate image extraction results for farmland in different environments, and all have universal practicability

Figure 4. Extraction results of green farmland navigation lines in different environments

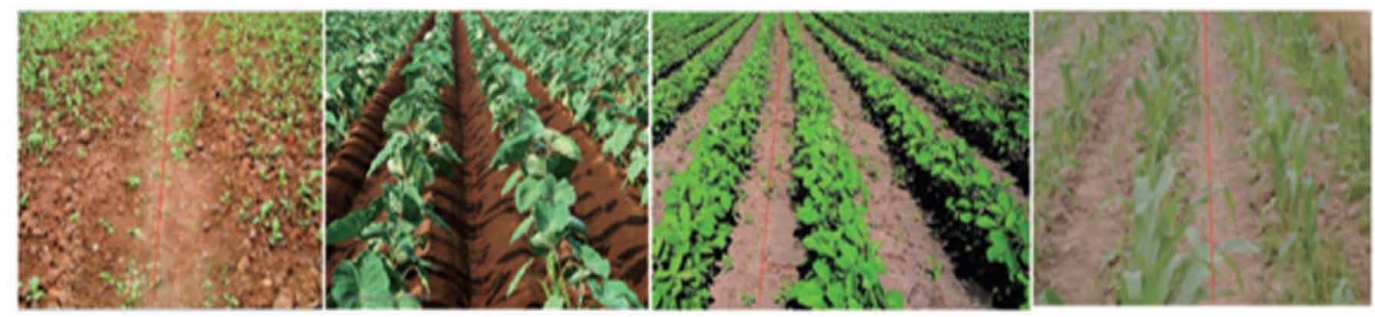

Figure 5. Extraction results of non-green farmland navigation lines in different environments

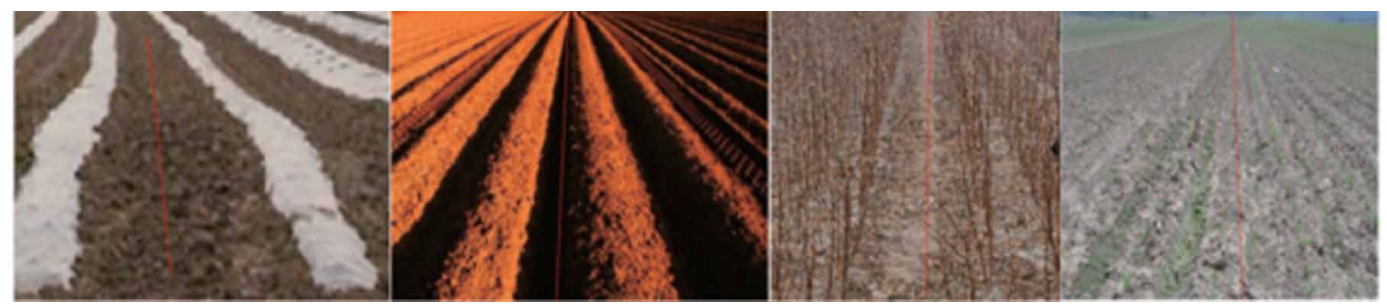


and high definition. But according to different weather conditions, the adaptability of various farmland navigation line algorithms in cloudy and sunny days has been verified to get a more accurate algorithm that adapts to the navigation line of agricultural machinery.

In the Matlab2010 (b) software environment, the above-mentioned algorithm and the traditional Hough transform method are used to calculate the time and efficiency of the navigation line imaging in 50 images. The navigation line can be seen in the comparison results of each stage the efficiency is shown in Table 1. The value of time consumed when converting based on dark primary color grayscale is 5 times the average consumption of the 2G-R-B super green model algorithm (Parker C, 1975). Among all the algorithms, Hough's algorithm is the most time-consuming. It can be seen that after this experiment is run in the $\mathrm{C}$ language environment, the algorithm is improved by at least 10 times (Patnaik A, Narayanamoorthi R, 2015). By using the Matlab 2010 software environment, which greatly meets the real-time requirements of image processing of farmland navigation lines. In summary, the algorithm in the above experiment is faster than the traditional Hough change, and fully meets the real-time and applicability of the navigation line test, and has little effect on the image results in different farmland environments. Therefore, these algorithms have an important position in the visual navigation system of small agricultural machinery robots, and these algorithms have a wide range of practical performance. To further understand the Figure 6. shows average time loss statistics graph.

Figure 7. demonstrates the evaluation of parameters of dissimilar algorithms. In Figure 8, the navigation line extraction results of different algorithms are compared with the proposed algorithm, SF algorithm, Hough transform algorithm, etc., and it is found that the red line represents the navigation line. Experiments show that the above algorithms can accurately extract the navigation lines from the images of the farmland in different environments (Yaghoubi S et.al. 2013). It can be seen from the data in Table 2 that the linear parameters of the four algorithms are compared. The maximum difference in slope is 1.8711 and the maximum difference in intercept is 672.3926 .

Table 1. Navigation line extraction time statistics table

\begin{tabular}{|c|c|c|c|c|}
\hline Method & Dark channel & 2G-R-B & Proposed method & Hough transformation \\
\hline Average time loss/s & 0.744 & 3.547 & $\mathbf{1 . 3 2 5}$ & 4.274 \\
\hline
\end{tabular}

Figure 6. Average time loss statistics graph

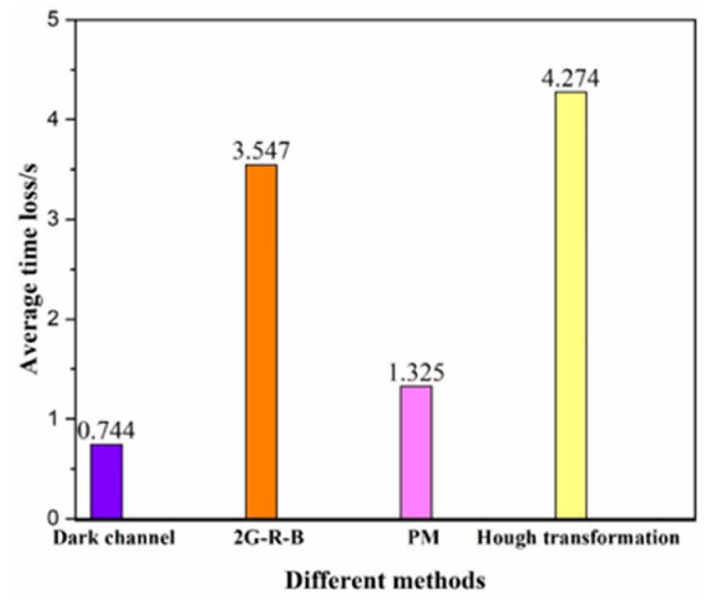


Figure 7. Evaluation of parameters of dissimilar algorithms

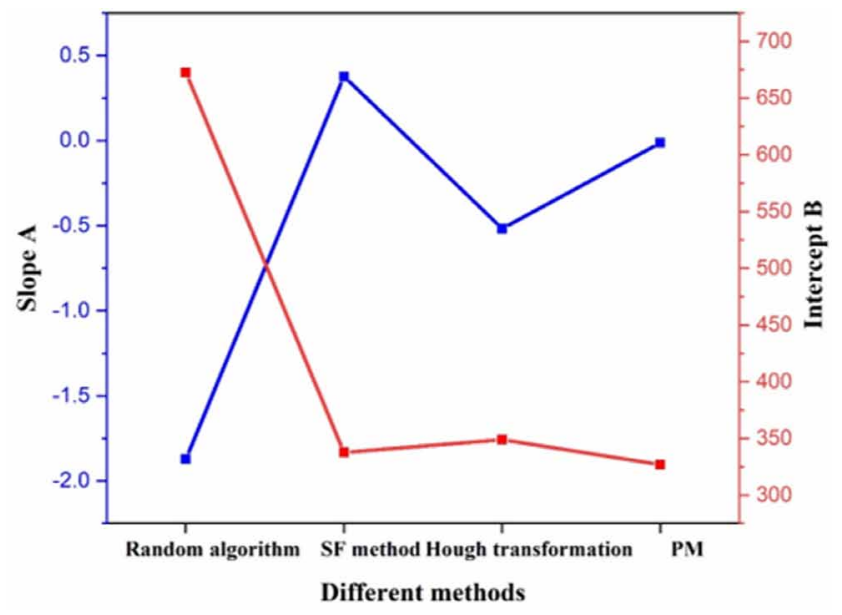

Figure 8. Navigation line extraction results of different algorithms (a) Proposed algorithm; (b) SF algorithm; (c) Hough transformation algorithm; (d) Random algorithm
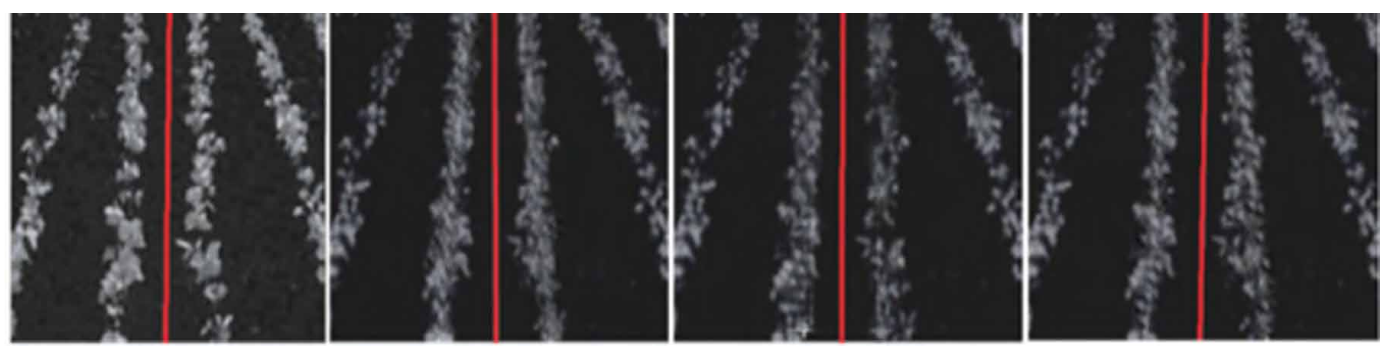

Table 2. Comparison of linear parameters of different algorithms

\begin{tabular}{|l|c|c|}
\hline \multicolumn{1}{|c|}{ Method } & Slope a & Intercept b \\
\hline Proposed Method(PM) & $\mathbf{- 0 . 0 1 3 4}$ & $\mathbf{3 2 7 . 0 2 2 2}$ \\
\hline SF Method & 0.3764 & 337.6437 \\
\hline Hough Transformation & -0.5168 & 348.9507 \\
\hline Random Algorithm & -1.8711 & 672.3926 \\
\hline
\end{tabular}

It can be seen from Table 2 that the linear parameters of the above algorithm are compared with The other three have the smallest difference compared to meet the precise requirements of the visual navigation line of the farm robot (Welte, 2016).To verify the stability and anti-interference performance of the above algorithm, targeted experiments were carried out on various interference environments such as a large amount of water environment, foggy weather, and vibration of agricultural machinery in the experimental environment (Vermesan and Friess, 2016). It is found that as shown in the experimental image in Figure, the grayscale image has been widely used in the dehazing algorithm, and the guideline image has a better effect (Wolfert et al., 2017). Therefore, the algorithms are shown in the proposed (a) and (b) images can be the images in cloudy and sunny environments are compared through the operating conditions of agricultural machinery. In figure 8, as shown in 
(c) and (d), after comparing the images of the test field in anhydrous and watery environments, the difference (Yoon B, Kim S, 2013). The algorithm navigation line extraction results found that the farmland underwater or no water environment has a low impact on the extraction results of the grayscale processing navigation line, but based on the dark primary color grayscale image processing, agricultural machinery vibration and foggy weather The environment is disturbing to the navigation line extraction algorithm(Wolfert et al., 2014).

\section{CONCLUSION}

The major problem of the super-green features of the color space model is selected for image grayscale and segmentation, which cannot be applied to crop navigation in a non-green environment. A new method of farmland image grayscale based on the dark primary color principle is proposed. The gray rate of dark primary color is much faster than that of 2 G-R-B. According to the characteristics of the position of the navigation line of farmland robot. A central navigation line extraction algorithm based on the vertical projection method is proposed. The algorithm is faster, less complex, and less computational, which not only improves the speed but also ensures accuracy. In a word, the proposed algorithm is based on the dark primary color of the farmland robot's visual navigation line. The tested and simulated proposed method achieves Intercept $b$ is equal to 327.0222 and slope $\mathrm{a}$ is equal to -0.0134 . The average time loss/s of the proposed method is 1.325 . This extraction has a good effect on green farmland environment, non-green farmland environment (such as farmland during the farming period, newly sprouted seedling farmland, low straw, and mature crop farmland, etc.), and farmland road environment navigation line extraction. Compared with other algorithms, the proposed algorithm can meet the needs of farmland robot visual navigation with more precision and faster speed, and wider applicability. 


\section{REFERENCES}

Chena, J., Qianga, H., Wua, J., Xua, G., Wanga, Z., \& Liua, X. (2020). Extracting the navigation path of a tomato-cucumber greenhouse robot based on a median point Hough transform. Computers and Electronics in Agriculture, 105472. 10.1016/j.compag.2020.105472

Cohen, J. (2009). Graph Twiddling in a MapReduce World. Computing in Science \& Engineering, 11(4), 29-41. doi:10.1109/MCSE.2009.120

Cole, J. B., Newman, S., Foertter, F., Aguilar, I., \& Coffey, M. (2012). Breeding and genetics symposium: Really big data: Processing and analysis of very large data sets1. Journal of Animal Science, 90(3), 723-733. doi:10.2527/jas.2011-4584 PMID:22100598

Cooper, M., van Eeuwijk, F. A., Hammer, G. L., Podlich, D. W., \& Messina, C. (2009). Modeling QTL for complex traits: Detection and context for plant breeding. Current Opinion in Plant Biology, 12(2), 231-240. doi:10.1016/j.pbi.2009.01.006 PMID:19282235

De Wagter, C., Ruijsink, R., Smeur, E. J. J., van Hecke, K. G., van Tienen, F., van der Horst, E., \& Remes, B. D. W. (2018). Design, control, and visual navigation of the DelftaCopter VTOL tail-sitter UAV. Journal of Field Robotics, 35(6), 937-960. doi:10.1002/rob.21789

Dong, Y., \& Itoh, T. (2012). Planar Ultra-Wideband Antennas in Ku- and K-Band for Pattern or Polarization Diversity Applications. IEEE Transactions on Antennas and Propagation, 60(6), 2886-2895. doi:10.1109/ TAP.2012.2194680

Dutta, R., Morshed, A., Aryal, J., D’Este, C., \& Das, A. (2014). Development of an intelligent environmental knowledge system for sustainable agricultural decision support. Environmental Modelling \& Software, 52, 264-272. doi:10.1016/j.envsoft.2013.10.004

El Bilali, H., \& Allahyari, M. S. (2018). Transition towards sustainability in agriculture and food systems: Role of information and communication technologies. Information Processing in Agriculture, 5(4), 456-464. doi:10.1016/j.inpa.2018.06.006

Elsheakh, D. N., Elsadek, H. A., Abdallah, E. A., Elhenawy, H., \& Iskander, M. F. (2009). Enhancement of Microstrip Monopole Antenna Bandwidth by Using EBG Structures. Antennas Wirel. Propag. Lett., 8, 959-962. doi:10.1109/LAWP.2009.2030375

Feng, H., Gao, N., Meng, Z., Chen, L., Li, Y., \& Guo, Y. (2018). Design and experiment of deep fertilizer applicator based on autonomous navigation for precise rowfollowing. Nongye Jixie Xuebao. Nongye Jixie Хиebao, 49(4), 60-67.

Garcia-Vico, A. M., Carmona, C. J., Gonzalez, P., \& del Jesus, M. J. (2018). MOEA-EFEP: Multi-Objective Evolutionary Algorithm for Extracting Fuzzy Emerging Patterns. IEEE Transactions on Fuzzy Systems, 26(5), 2861-2872. doi:10.1109/TFUZZ.2018.2814577

Guo, H., Wu, X., \& Li, N. (2018). Action Extraction in Continuous Unconstrained Video for Cloud-Based Intelligent Service Robot. IEEE Access: Practical Innovations, Open Solutions, 6, 33460-33471. doi:10.1109/ ACCESS.2018.2842088

Huang, Y., Chen, Z., Yu, T., Huang, X., \& Gu, X. (2018). Agricultural remote sensing big data: Management and applications. Journal of Integrative Agriculture, 17(9), 1915-1931. doi:10.1016/S2095-3119(17)61859-8

Ntaliani, M., \& Costopoulou, C. (2013). Adopting E-training and Living Labs for Collaborative Learning for Rural Public Agencies. JAI, 4. 10.17700/jai.2013.4.1.104

Parker, C., \& Fryer, J. D. (1975). Weed control problems causing major reduction in world food supplies. FAO Plant Protection Bulletin, 23, 83-95.

Patnaik, A., \& Narayanamoorthi, R. (2015). Weed removal in cultivated field by autonomous robot using LabVIEW. IEEE Int Conf on Innovations in Information Embedded and Communication Systems, (March), 1-5. doi:10.1109/ICIIECS.2015.7193168

Patrick, R., David, B., \& Peter, I. C. (2020). Vision-based guidance for robot navigation in agriculture. Proceedings - IEEE International Conference on Robotics and Automation, 1693-1698. doi:10.1109/ICRA.2014.6907079 
Pavan, W., Fraisse, C.W., \& Peres, N.A. (2011). Development of a webbased disease forecasting system for strawberries. Comput Electron Agric, 75, 169-175. .compag.2010.10.01310.1016/j

Rangarajan, A. K., Raja, P., \& Ruiz, M. P. (2020). Task-based agricultural mobile robots in arable farming: A review. Spanish Journal of Agricultural Research, 1-13. doi:10.5424/sjar/2017151-9573

Sharma, A., Ansari, M. D., \& Kumar, R. (2017). A comparative study of edge detectors in digital image processing. 4th International Conference on Signal Processing, Computing and Control (ISPCC), 246-250. doi:10.1109/ISPCC.2017.8269683

Sharma, A., \& Kumar, R. (2019). Performance comparison and detailed study of AODV, DSDV, DSR, TORA and OLSR routing protocols in ad hoc networks. 2016 Fourth International Conference on Parallel, Distributed and Grid Computing (PDGC), 732-736. doi:10.1109/PDGC.2016.7913218

Sharma, A., Singh, P. K., Sharma, A., \& Kumar, R. (2019). An efficient architecture for the accurate detection and monitoring of an event through the sky. Computer Communications, 148, 115-128. doi:10.1016/j.comcom.2019.09.009

Tarannum, N., Rhaman, K., Khan, S.A., \& Shakil, S.R. (2015). A Brief Overview and Systematic Approch for Using Agricultural Robot in Developing Countries. Academic Press.

Tien, J. M. (2013). Big Data: Unleashing information. Journal of Systems Science and Systems Engineering, 22(2), 127-151. doi:10.1007/s11518-013-5219-4

Tilman, D., Balzer, C., Hill, J., \& Befort, B. L. (2011). Global food demand and the sustainable intensification of agriculture. Proceedings of the National Academy of Sciences of the United States of America, 108(50), 20260-20264. doi:10.1073/pnas.1116437108 PMID:22106295

Torii, T. (2000). Research in autonomous agriculture vehicles in Japan. Computers and Electronics in Agriculture, 25(1-2), 133-153. doi:10.1016/S0168-1699(99)00060-5

Vermesan, O., \& Friess, P. (2016). Digitising the Industry - Internet of Things Connecting the Physical. Digital and Virtual Worlds. doi:10.13052/rp-9788793379824

Welte, J.T. (n.d.). A Farm Management Information System With Task-Specific, Collaborative Mobile Apps And Cloud Storage Services. Academic Press.

Wolfert, S., Ge, L., Verdouw, C., \& Bogaardt, M.-J. (2017). Big Data in Smart Farming - A review. Agricultural Systems, 153, 69-80. doi:10.1016/j.agsy.2017.01.023

Wolfert, S., Goense, D., \& Sorensen, C. A. G. (2014). A Future Internet Collaboration Platform for Safe and Healthy Food from Farm to Fork. In 2014 Annual SRII Global Conference. Presented at the 2014 Annual SRII Global Conference (SRII). IEEE. doi:10.1109/SRII.2014.47

Wu, X., Zhu, X., Wu, G.-Q., \& Ding, W. (2014). Data mining with big data. IEEE Transactions on Knowledge and Data Engineering, 26(1), 97-107. doi:10.1109/TKDE.2013.109

Yaghoubi, S., Akbarzadeh, N. A., Bazargani, S. S., Bazargani, S. S., Bamizan, M., \& Asl, M. I. (2013). Autonomous robots for agricultural tasks and farm assignment and future trends in agro robots. Int $J$ Mech Mechatron Eng, 13, 1-6.

Ye, Q., Qu, C., \& Zhang, Y. (n.d.). Human motion analysis based on extraction of skeleton and dynamic time warping algorithm using RGBD camera. Academic Press.

Yoon, B., \& Kim, S. (2013). Design of Paddy weeding robot. IEEE Int Symp on Robotics, (October), 1-2.

Zhang, M., Lu, X., Tao, J., Yin, W., \& Feng, X. (2016). Design and experiment of the automatic guidance control system in agriculture vehicle. Nongye Jixie Xuebao. Nongye Jixie Xuebao, 47, $42-47$.

Zhang, Q., Shaojie, M. E., \& Li, B. (2017). A visual navigation algorithm for paddy field weeding robot based on image understanding. Computers and Electronics in Agriculture, 143, 66-78. doi:10.1016/j.compag.2017.09.008

Zhao, Y. (2019). Design and Application of an Adaptive Slow Feature Extraction Algorithm for Natural Images Based on Visual Invariance. Traitement du Signal. doi:10.18280/ts.360302

Zhu, S., Liu, D., Liu, Y., \& Cui, P. (2019). Observability-based visual navigation using landmarks measuring angle for pinpoint landing. Acta Astronautica, 155, 313-324. doi:10.1016/j.actaastro.2018.10.011 